\title{
Self-gravitating bosons at nonzero temperature
}

\author{
Neven Bilić ${ }^{*}$ and Hrvoje Nikolić \\ Theoretical Physics Division, Rudjer Bošković Institute, \\ P.O.B. 180, 10002 Zagreb, Croatia \\ *bilic@thphys.irb.hr
}

October 29, 2018

\begin{abstract}
A system of charged bosons at finite temperature and chemical potential is studied in a general-relativistic framework. We assume that the boson fields interact only gravitationally. At sufficiently low temperature the system exists in two phases: the gas and the condensate. By studying the condensation process numerically we determine the critical temperature $T_{\mathrm{c}}$ at which the condensate emerges. As the temperature decreases, the system eventually settles down in the ground state of a cold boson star. PACS: 04.40.-b, 05.30.Jp, 11.10.Wx
\end{abstract}

Keywords: self-gravitating bosons, boson star, Bose-Einstein condensation

\section{Introduction}

Bose-Einstein condensation is an extensively studied subject of interest to almost all branches of physics. It is probably the simplest example of critical phenomena since it exists theoretically even in noninteracting systems [1, 2, 3]. In an interacting theory containing complex scalar fields, Bose-Einstein condensation becomes a highly nontrivial phenomenon. In self-interacting scalar theories there are cases, such as $\phi^{4}$ theory, where a homogeneous condensate is a stable ground state. In some cases, the Bose-Einstein condensate at zero temperature exists in the form of stable nontopological solitons known as Q-balls [4, 5]. Another interesting example is a scalar field coupled to gravity. The ground state of a condensed cloud of charged bosons of mass $m$, interacting only gravitationally and having a total mass $M$ below a certain limit of the order $M_{\mathrm{Pl}}^{2} / m$, is a stable spherically symmetric configuration [6] which is usually referred to as mini-soliton star [7] or boson star [8]. The gravitational collapse of boson stars is prevented by Heisenberg's uncertainty principle. Seidel and Suen have demonstrated that boson stars may be formed through a dissipationless mechanism, called gravitational cooling [9]. Boson stars have recently attracted much attention as they may well be candidates for nonbaryonic dark matter [10].

In this paper we study the thermodynamics of boson stars and its relation to the BoseEinstein condensation in the framework of general relativity. Some aspects of boson stars at finite temperature have been studied in Newtonian gravity [11]. Our approach is similar 
in spirit to the analysis of Q-ball thermodynamics by Laine and Shaposhnikov [12]. We will consider a canonical ensemble of charged bosons and study its properties near the critical temperature at which the Bose-Einstein condensate forms.

We organize the paper as follows: In Section 2 we discuss the grand canonical ensemble of gravitating bosons within a general-relativistic framework. In Section 3 the free energy is derived for a canonical system of gravitating bosons. In Section t we discuss the equations that describe a boson star at finite temperature and their numerical integration. Numerical results are presented in Section 5. Conclusions are drawn in Section 6.

\section{Grand canonical ensemble}

In this section we derive the grand canonical partition function for a system of self-gravitating charged bosons contained in a sphere of large radius $R$ in equilibrium at finite temperature $T=1 / \beta$. In a grand canonical ensemble we introduce the chemical potential $\mu$ associated to the conserved particle number $N$. The partition function is given by

$$
Z=\operatorname{Tr} e^{-\beta(H-\mu N)}=\int[d g][d \Phi]\left[d \Phi^{*}\right] e^{-S_{\mathrm{g}}-S_{\mathrm{KG}}},
$$

with the Euclidean actions $S_{\mathrm{g}}$ and $S_{\mathrm{KG}}$ for the gravitational and the Klein-Gordon fields, respectively. The gravitational part may be put in the form 13, 14

$$
S_{\mathrm{g}}=-\frac{1}{16 \pi} \int_{Y} d^{4} x \sqrt{g} \mathcal{R}-\frac{1}{8 \pi} \int_{\partial Y} d^{3} x \sqrt{h}\left(K-K_{0}\right),
$$

where $\mathcal{R}$ is the curvature scalar, $h$ is the determinant of the induced metric on the boundary, and $K-K_{0}$ is the difference in the trace of the second fundamental form of the boundary $\partial Y$ in the metric $g$ and the flat metric. The boundary is a timelike tube of radius $R$ which is periodically identified in the imaginary time direction with period $\beta$. Thus, the functional integration assumes the periodicity in imaginary time and the asymptotic flatness of the metric fields. The matter action is given by

$$
S_{\mathrm{KG}}=\int_{Y} d^{4} x \sqrt{g}\left(g^{\mu \nu} \partial_{\mu} \Phi^{*} \partial_{\nu} \Phi+m^{2}|\Phi|^{2}\right),
$$

with the replacement of the Euclidean time derivative [15

$$
\frac{\partial}{\partial \tau} \rightarrow \frac{\partial}{\partial \tau} \pm \mu
$$

where the + or - sign is taken when the derivative acts on $\Phi^{*}$ or $\Phi$, respectively. The path integral is taken over asymptotically vanishing fields which are periodic in imaginary time $\tau$ with period $\beta$. The dominant contribution to the path integral comes from metrics and matter fields, which are near the classical fields $g_{\mu \nu}, \Phi^{*}$, and $\Phi$. The classical fields extremize the action, i.e., they are solutions to the classical field equations. The background metric generated by the mass distribution is static, spherically symmetric, and asymptotically flat, i.e.,

$$
d s^{2}=e^{\nu(r)} d t^{2}-e^{\lambda(r)} d r^{2}-r^{2}\left(d \vartheta^{2}+\sin ^{2} \vartheta d \varphi^{2}\right)
$$


Substitution $t=-i \tau$ converts this into a positive definite metric with the Euclidean signature,

$$
d s_{\mathrm{E}}^{2}=e^{\nu(r)} d \tau^{2}+e^{\lambda(r)} d r^{2}+r^{2}\left(d \vartheta^{2}+\sin ^{2} \vartheta d \varphi^{2}\right)
$$

In order to extract the classical contribution, we decompose $\Phi$ as

$$
\Phi(x)=\phi(x)+\psi(x)
$$

where $\phi$ is a solution to the Klein-Gordon equation which we will call condensate and $\psi$ describes quantum and thermal fluctuations around $\phi$. If we neglect quantum fluctuations of the metric, the partition function factorizes as

$$
Z=Z_{\mathrm{g}} Z_{\mathrm{cd}} \int[d \psi]\left[d \psi^{*}\right] e^{-S_{\mathrm{KG}}\left[\psi, \psi^{*}\right]},
$$

where $Z_{\mathrm{g}}=e^{-S_{\mathrm{g}}}$ and $Z_{\mathrm{cd}}=e^{-S_{\mathrm{cd}}}$ represent the saddle-point gravitational and condensate contributions, respectively. The condensate action is given by

$$
S_{\mathrm{cd}}=\int_{Y} d^{4} x \sqrt{g}\left(g^{\mu \nu} \partial_{\mu} \phi^{*} \partial_{\nu} \phi+m^{2}|\phi|^{2}\right) .
$$

Variation of $S_{\text {cd }}$ with respect to $\phi^{*}$ yields a Klein-Gordon equation in which the time derivatives are replaced by (4). With the metric (6) and the usual ansatz for static solutions

$$
\begin{aligned}
& \phi(t, r)=\frac{1}{\sqrt{2}} e^{-i \omega^{\prime} t} \varphi(r)=\frac{1}{\sqrt{2}} e^{-\omega^{\prime} \tau} \varphi(r), \\
& \phi^{*}(t, r)=\frac{1}{\sqrt{2}} e^{i \omega^{\prime} t} \varphi(r)=\frac{1}{\sqrt{2}} e^{\omega^{\prime} \tau} \varphi(r),
\end{aligned}
$$

we obtain the static Klein-Gordon equation

$$
\frac{d^{2} \varphi}{d r^{2}}+\frac{1}{2}\left(\frac{d \nu}{d r}-\frac{d \lambda}{d r}+\frac{4}{r}\right) \frac{d \varphi}{d r}+e^{\lambda}\left(\omega^{2} e^{-\nu}-m^{2}\right) \varphi=0
$$

where $\omega=\omega^{\prime}+\mu$. Regular, asymptotically vanishing solutions to equation (12) coupled with Einstein field equations are solitons which describe the well-known boson stars [7, 8] at zero temperature. Our aim is to extend and analyze the corresponding solutions at finite temperature.

Ignoring for the moment the periodicity condition, the ansatz $(10,11)$ gives the condensate contribution to the partition function

$$
\ln Z_{\mathrm{cd}}=\frac{1}{2} \beta \int_{\Sigma} d^{3} x \sqrt{g}\left[g^{00} \omega^{2} \varphi^{2}-g^{i i}\left(\partial_{i} \varphi\right)^{2}-m^{2} \varphi^{2}\right],
$$

where $\Sigma$ is a spacelike hypersurface that contains the condensate. Using this expression we find the net number of particles in the condensate

$$
N_{\mathrm{cd}}=\frac{1}{\beta} \frac{\partial \ln Z_{\mathrm{cd}}}{\partial \mu}=\int_{\Sigma} d^{3} x \sqrt{g} g^{00} \omega \varphi^{2} .
$$


Alternatively, we define the particle number in a covariant way as

$$
N_{\mathrm{cd}}=\int_{\Sigma} n_{\mathrm{cd}} u^{\mu} d \Sigma_{\mu}=\int_{\Sigma} d^{3} x \sqrt{g_{(3)}} n_{\mathrm{cd}},
$$

where $n_{\mathrm{cd}}$ is the particle-number density in the condensate and $g_{(3)}=\operatorname{det}\left(g_{i j}\right), i, j=1,2,3$. Here we have used the fluid four-velocity $u^{\mu}$ the components of which in the comoving frame are

$$
u^{\mu}=\frac{\delta_{0}^{\mu}}{\sqrt{g_{00}}} ; \quad u_{\mu}=\frac{g_{\mu 0}}{\sqrt{g_{00}}} .
$$

Therefore, we identify the particle-number density due to the condensate as

$$
n_{\mathrm{cd}}=\sqrt{g^{00}} \omega \varphi^{2} .
$$

It may be easily verified that the four-vector $j^{\mu}=n_{\mathrm{cd}} u^{\mu}$ coincides with the current defined as

$$
j^{\mu}=g^{\mu \nu}\left(\phi \partial_{\mu} \phi^{*}-\phi^{*} \partial_{\mu} \phi\right) .
$$

In order to satisfy the periodicity condition, i.e., $\phi(\beta)=\phi(0)$, we must set $\omega^{\prime}=0$. This in turn implies $\omega=\mu$. In other words, as it was pointed out by Laine and Shaposhnikov [12], if there exist soliton solutions with some values of $\omega$, these solutions are saddle points of the Euclidean path integral at $\mu=\omega$. In the absence of gravity, a saddle-point solution is a homogeneous Bose-Einstein condensate of a free relativistic boson gas.

Next, we calculate the thermal contribution to $Z$ starting from the expression

$$
Z_{\mathrm{th}}=\int[d \psi]\left[d \psi^{*}\right] e^{-S_{\mathrm{KG}}\left[\psi, \psi^{*}\right]},
$$

with

$$
S_{\mathrm{KG}}=\int_{Y} d^{4} x \sqrt{g}\left(g^{\mu \nu} \partial_{\mu} \psi^{*} \partial_{\nu} \psi+m^{2}|\psi|^{2}\right) .
$$

The metric in equilibrium is static, i.e., $g_{\mu \nu}$ is independent of $\tau$ and $g_{0 i}=0$. Hence, the determinant of $g_{\mu \nu}$ factorizes as $g=g_{00} g_{(3)}$. By making use of the substitution $\bar{\tau}=\tau \sqrt{g_{00}}$ we obtain

$$
S_{\mathrm{KG}}=\int d^{3} x \sqrt{g_{(3)}} \int_{0}^{\bar{\beta}} d \bar{\tau} \mathcal{L}(\bar{\tau}, x)
$$

where

$$
\mathcal{L}(\bar{\tau}, x)=\left(\partial_{\bar{\tau}}+\bar{\mu}\right) \psi^{*}\left(\partial_{\bar{\tau}}-\bar{\mu}\right) \psi+g^{i j} \partial_{i} \psi^{*} \partial_{j} \psi+m^{2}|\psi|^{2} .
$$

Here we have defined the local chemical potential and the inverse local temperature as

$$
\bar{\mu}=\mu / \sqrt{g_{00}} ; \quad \bar{\beta}=\beta \sqrt{g_{00}} .
$$

These expressions are nothing but the well-known Tolman conditions for chemical and thermal equilibrium in a gravitational field [16, 17, 18]. Thus, the parameters $\beta$ and $\mu$ are interpreted as, respectively the values of the inverse temperature and chemical potential at infinity [19]. In a close neighborhood $\Sigma_{0}$ of a space point $X$ we introduce local spatial coordinates $y=f_{X}(x)$ such that

$$
g^{i j} \frac{\partial \psi^{*}}{\partial x_{i}} \frac{\partial \psi}{\partial x_{j}}=\delta^{i j} \frac{\partial \psi_{X}^{*}}{\partial y_{i}} \frac{\partial \psi_{X}}{\partial y_{j}},
$$


where $\psi_{X}(y)=\psi\left(f_{X}^{-1}(y)\right)$. The Lagrangian (22) is now locally represented by the Euclidean flat-space Lagrangian

$$
\mathcal{L}(\bar{\tau}, X, y)=\left(\frac{\partial \psi_{X}^{*}}{\partial \bar{\tau}}+\bar{\mu} \psi_{X}^{*}\right)\left(\frac{\partial \psi_{X}}{\partial \bar{\tau}}-\bar{\mu} \psi_{X}\right)+\frac{\partial \psi_{X}^{*}}{\partial y^{i}} \frac{\partial \psi_{X}}{\partial y_{i}}+m^{2}\left|\psi_{X}\right|^{2} .
$$

By integrating $\mathcal{L}(\bar{\tau}, X, y)$ over $y$ we define

$$
\mathcal{L}(\bar{\tau}, X)=\frac{1}{V_{0}} \int_{\Sigma_{0}} d^{3} y \mathcal{L}(\bar{\tau}, X, y)
$$

where $V_{0}$ is the volume of $\Sigma_{0}$. Using the adiabatic approximation $\mathcal{L}(\bar{\tau}, x) \approx \mathcal{L}(\bar{\tau}, X)$, which holds for sufficiently small $V_{0}$, we obtain

$$
S_{\mathrm{KG}}=\int d^{3} X \sqrt{g_{(3)}(X)} \int_{0}^{\bar{\beta}} d \bar{\tau} \frac{1}{V_{0}} \int_{\Sigma_{0}} d^{3} y \mathcal{L}(\bar{\tau}, X, y)
$$

Now, the partition function (19) may be factorized as

$$
Z_{\mathrm{th}}=\prod_{x} Z_{x}
$$

where

$$
Z_{x}=\int[d \psi]\left[d \psi^{*}\right] \exp \left[-\int d^{4} y d^{4} y^{\prime} \psi^{*}(y) K_{x}\left(y, y^{\prime}\right) \psi\left(y^{\prime}\right)\right]
$$

with

$$
\left.K_{x}\left(y, y^{\prime}\right)=V_{0}^{-1} \sqrt{g_{(3)}(x)}\left[\left(\partial_{\bar{\tau}}-\bar{\mu}\right)^{2}+\nabla_{y}^{2}\right)\right] \delta^{(4)}\left(y-y^{\prime}\right) .
$$

Assuming that $V_{0}$, although small, is still macroscopic and that the thermodynamic limit may be applied to each $Z_{x}$, the standard functional integration technique [15] gives

$$
\begin{aligned}
\ln Z_{\mathrm{th}} & =\int d^{3} x \operatorname{Tr} \ln K_{x}^{-1} \\
& =-\int d^{3} x \sqrt{g_{(3)}} \int \frac{d^{3} q}{(2 \pi)^{3}}\left[\ln \left(1-e^{-\bar{\beta}(E-\bar{\mu})}\right)+\ln \left(1-e^{-\bar{\beta}(E+\bar{\mu})}\right)\right],
\end{aligned}
$$

where $E=\sqrt{q^{2}+m^{2}}$. This expression may be regarded as a proper volume integral of the local partition function $\ln z(x)$

$$
\ln z=-\int \frac{d^{3} q}{(2 \pi)^{3}}\left[\ln \left(1-e^{-\bar{\beta}(E-\bar{\mu})}\right)+\ln \left(1-e^{-\bar{\beta}(E+\bar{\mu})}\right)\right]
$$

from which the pressure, energy density, particle-number density, and entropy density may be derived in the usual way:

$$
\begin{gathered}
p_{\mathrm{th}}=\frac{1}{\bar{\beta}} \ln z, \\
\rho_{\mathrm{th}}=-\left.\frac{\partial}{\partial \bar{\beta}} \ln z\right|_{\bar{\beta} \bar{\mu}},
\end{gathered}
$$




$$
\begin{gathered}
n_{\mathrm{th}}=\frac{1}{\bar{\beta}} \frac{\partial}{\partial \bar{\mu}} \ln z, \\
\sigma=\bar{\beta}\left(p_{\mathrm{th}}+\rho_{\mathrm{th}}-\bar{\mu} n_{\mathrm{th}}\right) .
\end{gathered}
$$

These expressions together with (32) yield the well-known parametric representation of the equation of state of a relativistic Bose gas in curved space. This equation of state is a special case of a more general expression derived from the relativistic kinetic theory [18].

The gravitational part of the partition function may be calculated from (2) with help of Einstein field equations. Using the result of Gibbons and Hawking for the surface term [13], we obtain

$$
\ln Z_{\mathrm{g}}=-\beta M+\int_{Y} d^{4} x \sqrt{g} T_{0}^{0},
$$

where $M$ is the total mass and $T_{\mu}^{\nu}$ is the energy-momentum tensor of the Klein-Gordon field averaged with respect to the partition function (8). The energy-momentum tensor of the Klein-Gordon field can be obtained by variation of the the Euclidean Klein-Gordon action with respect to the metric

$$
T_{\mu \nu}(\Phi)=-\frac{2}{\sqrt{g}} \frac{\delta S_{\mathrm{KG}}}{\delta g^{\mu \nu}}
$$

From (3) with (4) we find

$$
T_{\mu \nu}(\Phi)=-\partial_{\mu} \Phi^{*} \partial_{\nu} \Phi-\partial_{\nu} \Phi^{*} \partial_{\mu} \Phi+g_{\mu \nu}\left(g^{\rho \sigma} \partial_{\rho} \Phi^{*} \partial_{\sigma} \Phi+m^{2}|\Phi|^{2}\right) .
$$

The averaged energy momentum tensor may be split up into two parts:

$$
T_{\mu \nu}=T_{\mu \nu}(\varphi)+T_{\text {th } \mu \nu}
$$

where the first term on the right-hand side is the classical part which comes from the condensate and the second term represents the thermal and quantum fluctuations. By making use of (10) and (11) we obtain the condensate contribution to $T_{0}^{0}$ :

$$
\rho_{\mathrm{cd}}=T_{0}^{0}(\varphi)=\frac{1}{2}\left(g^{00} \omega^{2} \varphi^{2}+g^{i i}\left(\partial_{i} \varphi\right)^{2}+m^{2} \varphi^{2}\right) .
$$

It may be shown that the thermal part is of the form which characterizes a perfect fluid:

$$
T_{\text {th } \mu \nu}=\left(\rho_{\text {th }}+p_{\text {th }}\right) u_{\mu} u_{\nu}-p_{\text {th }} g_{\mu \nu},
$$

where the thermal pressure and energy density are given by (33) and (34), respectively. Putting the condensate (13), the thermal (31), and the gravitational (37) contributions together, we find the total thermodynamical potential as

$$
\Omega(\beta, \mu)=-\frac{1}{\beta} \ln Z=M-\int_{\Sigma} d^{3} x \sqrt{g}\left[g^{00} \omega^{2} \varphi^{2}+p_{\mathrm{th}}+\rho_{\mathrm{th}}\right] .
$$




\section{Canonical ensemble}

Now consider a system of self-gravitating charged bosons with the net number of particles (number of particles minus number of antiparticles) $N$ contained in a two-dimensional sphere of large radius $R$ in equilibrium at finite temperature $T=1 / \beta$. In a canonical ensemble, instead of the chemical potential $\mu$ we fix the particle number which is the sum of the condensate and thermal contributions. Thus, a canonical ensemble is subject to the constraint

$$
\int_{\Sigma} d^{3} x \sqrt{g_{(3)}}\left(n_{\mathrm{cd}}+n_{\mathrm{th}}\right)=N
$$

where $n_{\text {cd }}$ is given by (17) and $n_{\text {th }}$ by (35) with (32).

The free energy of a canonical ensemble may be derived from the grand-canonical partition function with the help of the Legendre transform

$$
F(\beta, N)=\Omega(\beta, \mu)+\mu N
$$

The quantity $\mu$ in this expression is an implicit function of $N$ and $T$, such that for given $N$ and $T$ the constraint (44) is satisfied [20]. From (43) and (44) with (17) it follows

$$
F=M-(\omega-\mu) \int_{\Sigma} d^{3} x \sqrt{g} g^{00} \omega \varphi^{2}-\int_{\Sigma} d^{3} x \sqrt{g}\left(p_{\mathrm{th}}+\rho_{\mathrm{th}}-\bar{\mu} n_{\mathrm{th}}\right) .
$$

If $\omega=\mu$, the second term on the right-hand side vanishes and the free energy may be expressed in the familiar form

$$
F=M-T S
$$

where the total entropy $S$ is defined as a proper volume integral

$$
S=\int_{\Sigma} \sigma u^{\mu} d \Sigma_{\mu}=\int_{\Sigma} d^{3} x \sqrt{g_{(3)}} \sigma
$$

over the entropy density $\sigma$ given by (36).

\section{Numerical integration}

Given the temperature at infinity $T$, the radius of the sphere $R$, and the particle number $N$, we have to solve a set of self-consistency equations consisting of the Klein-Gordon equation (12) and Einstein field equations:

$$
\begin{aligned}
& \frac{d \nu}{d r}=8 \pi\left(p_{\mathrm{cd}}+p_{\mathrm{th}}\right) r e^{\lambda}+\frac{1}{r}\left(e^{\lambda}-1\right), \\
& \frac{d \lambda}{d r}=8 \pi\left(\rho_{\mathrm{cd}}+\rho_{\mathrm{th}}\right) r e^{\lambda}-\frac{1}{r}\left(e^{\lambda}-1\right),
\end{aligned}
$$

where the thermal pressure and energy density are given by (33) and (34), respectively, the condensate density $\rho_{\mathrm{cd}}$ is given by (41) and the radial pressure of the condensate by

$$
p_{\mathrm{cd}}=-T_{r}^{r}(\varphi)=\frac{1}{2}\left(g^{00} \omega^{2} \varphi^{2}+g^{i i}\left(\partial_{i} \varphi\right)^{2}-m^{2} \varphi^{2}\right) .
$$


In addition, we impose the constraint (44) which fixes the chemical potential $\mu$ at infinity. For numerical convenience, let us introduce the substitutions

$$
\begin{aligned}
e^{\nu(r)} & =\frac{\omega^{2}}{m^{2}} \frac{1}{\chi(r)+1}, \\
e^{\lambda(r)} & =\frac{1}{1-2 \mathcal{M}(r) / r}
\end{aligned}
$$

and new dimensionless parameters

$$
\alpha=\frac{\mu}{T}, \quad \gamma=\frac{\omega}{\mu}, \quad \eta=\frac{m^{2}}{M_{\mathrm{Pl}}^{2}},
$$

where $M_{\mathrm{Pl}}=\sqrt{\hbar c / G}$ denotes the Planck mass. Furthermore, we choose appropriate length and mass scales such that the length is measured in units of $1 / m$, and the mass in units of $M_{\mathrm{Pl}}^{2} / m$. In this way, all physical quantities appearing in our equations become dimensionless and equations (12), (49), and (50) now read

$$
\begin{gathered}
\frac{d^{2} \varphi}{d r^{2}}+\left(\frac{2 \mathcal{M}-4 \pi r^{3}(\tilde{\rho}-\tilde{p})}{r-2 \mathcal{M}}+2\right) \frac{1}{r} \frac{d \varphi}{d r}+\frac{r}{r-2 \mathcal{M}} \chi \varphi=0 \\
\frac{d \mathcal{M}}{d r}=4 \pi r^{2} \tilde{\rho} \\
\frac{d \chi}{d r}=-2(\chi+1) \frac{\mathcal{M}+4 \pi r^{3} \tilde{p}}{r(r-2 \mathcal{M})}
\end{gathered}
$$

To these three equations we add

$$
\frac{d \mathcal{N}}{d r}=4 \pi r^{2}(1-2 \mathcal{M} / r)^{-1 / 2} \tilde{n}
$$

imposing the particle-number constraint as a condition at the boundary:

$$
\mathcal{N}(R)=N
$$

In equations (55)-(58) we have used the abbreviations

$$
\begin{gathered}
\tilde{\rho}=\frac{\rho}{m^{2} M_{\mathrm{Pl}}^{2}}=\frac{1}{2}\left[(\chi+2) \varphi^{2}+\frac{r-2 \mathcal{M}}{r}\left(\frac{d \varphi}{d r}\right)^{2}\right]+\eta \tilde{\rho}_{\mathrm{th}}, \\
\tilde{p}=\frac{p}{m^{2} M_{\mathrm{Pl}}^{2}}=\frac{1}{2}\left[\chi \varphi^{2}+\frac{r-2 \mathcal{M}}{r}\left(\frac{d \varphi}{d r}\right)^{2}\right]+\eta \tilde{p}_{\mathrm{th}}, \\
\tilde{n}=\frac{n}{m M_{\mathrm{Pl}}^{2}}=\sqrt{\chi+1} \varphi^{2}+\eta \tilde{n}_{\mathrm{th}},
\end{gathered}
$$

where the dimensionless quantities $\tilde{\rho}_{\mathrm{th}}, \tilde{p}_{\mathrm{th}}$, and $\tilde{n}_{\mathrm{th}}$ are derived from (32)-(35):

$$
\tilde{\rho}_{\mathrm{th}}=\frac{\rho_{\mathrm{th}}}{m^{4}}=\frac{1}{2 \pi^{2}} \int_{0}^{\infty} d y y^{2} \sqrt{1+y^{2}}\left(n_{+}+n_{-}\right)
$$




$$
\begin{gathered}
\tilde{p}_{\mathrm{th}}=\frac{p_{\mathrm{th}}}{m^{4}}=\frac{1}{6 \pi^{2}} \int_{0}^{\infty} d y \frac{y^{4}}{\sqrt{1+y^{2}}}\left(n_{+}+n_{-}\right), \\
\tilde{n}_{\mathrm{th}}=\frac{n_{\mathrm{th}}}{m^{3}}=\frac{1}{2 \pi^{2}} \int_{0}^{\infty} d y y^{2}\left(n_{+}-n_{-}\right) \\
n_{ \pm}=\frac{1}{\exp \left[\alpha\left(\gamma \sqrt{\left(1+y^{2}\right) /(\chi+1)} \mp 1\right)\right]-1} .
\end{gathered}
$$

In equations (55)-(66) the radial coordinate $r$, the enclosed mass $\mathcal{M}$, and the particle number $N$ are measured in units of $1 / m, m / \eta, 1 / \eta$, respectively, and the scalar field $\varphi$ in units of $M_{\mathrm{Pl}}$.

It is evident from (60)-(66) that the thermal contribution to the local quantities is suppressed by the factor $\eta$, of the order of $10^{-38}$ for a boson mass of the order of $1 \mathrm{GeV}$. However, the contributions of the condensate and the thermal part to the global quantities, such as $M, N$, or $F$, may be of the same order of magnitude provided the volume of the system is sufficiently large. Indeed, the radius $R$ is much larger than the radial extent of the condensate, and, as we shall shortly demonstrate, the physically reasonable choice of $R$ makes the condensate and the thermal contributions comparable in magnitude. We shall also see that if we take the limit of vanishing $G$ keeping the central density fixed, we recover the free boson gas in which the densities of the condensate and the gas are homogeneous and comparable in magnitude for the temperatures above zero and below the critical temperature.

Equations (55)-(58) should be integrated with boundary conditions dictated by physics. To avoid singularities at the origin, we take

$$
\mathcal{M}(0)=0 ; \quad \mathcal{N}(0)=0 ;\left.\quad \frac{d \varphi}{d r}\right|_{r=0}=0
$$

The initial value of the scalar field $\varphi_{0} \equiv \varphi(0)$ at $r=0$ is arbitrary but it can be taken to be positive without loss of generality. The metric field at the origin $\chi_{0} \equiv \chi(0)$ is restricted to

$$
-1<\chi_{0} \leq \gamma^{2}-1
$$

because of the requirements that the metric should be positive and that the Bose-Einstein distribution (66) should be nonnegative everywhere. However, $\varphi_{0}$ and $\chi_{0}$ must be simultaneously tuned in order to satisfy the constraint (59) and to provide the correct asymptotic behavior $\varphi(r) \rightarrow 0$ as $r \rightarrow \infty$. The condition that the field $\varphi$ should vanish asymptotically is necessary in order that the condensate be either a soliton or absent. A homogeneous condensate is excluded for $G \neq 0$ owing to our assumption that the metric should be asymptotically flat.

Once we have found a solution on the interval $(0, R)$ for arbitrarily fixed parameters $\alpha$ and $\gamma$ with the correctly chosen initial conditions at $r=0$, we can determine the unknown physical parameters $\omega, \mu$, and $T$ from the boundary conditions at $r=R$. We cut off the matter from $R$ to infinity and join the interior solution onto the empty space Schwarzschild exterior solution

$$
\chi(r)=\frac{\omega^{2}}{m^{2}}\left(1-\frac{2 M}{r}\right)^{-1}-1 .
$$


Combining this equation with the numerically found interior solution at $r=R$, we obtain $\omega$ which then, together with (54), fixes the chemical potential $\mu$ and the temperature $T$ at infinity.

Consider first the condensate at zero temperature. We know that a zero-node soliton solution exist for any particle number $N$ below $N_{\max }=0.653$ [7]. This maximal value, known as the Kaup limit [6], corresponds to the initial values $\varphi_{0}=0.0764, \chi_{0}=0.545$, and the frequency $\omega=0.853 \mathrm{~m}$. Lower $\varphi_{0}$ lead to lower $N$ and larger $\omega$. In the limit $\varphi_{0} \rightarrow 0$, one approaches the Newtonian regime in which $\omega$ approaches the limiting value $m$. It may be easily shown that in the Newtonian limit there exist a mass-radius relationship of the form

$$
M R_{0}=\operatorname{const} \frac{M_{\mathrm{Pl}}^{2}}{m^{2}}
$$

where the radius $R_{0}$ of the boson star may be conveniently defined, e.g., as the radius of a ball of mass $M$ with constant density equal to the central density $\rho(0)=\left(1+\chi_{0} / 2\right) \varphi_{0}^{2} m^{2} M_{\mathrm{Pl}}^{2}$. Other definitions are possible [7] and lead to the same qualitative conclusions. Relation (70) enables us to analyze the fate of the boson star in the limit $G \rightarrow 0$. In that limit, we keep a certain physical quantity fixed, e.g., the particle number $N$, or the central density $\rho(0)$. In both cases, the radius $R_{0}$ increases with decreasing $G$ and becomes infinite for $G=0$. For example, if $\rho(0)$ is kept fixed, $R_{0}$ grows as $G^{-1 / 4}$ as a consequence of (70). This situation is illustrated in Fig. 1 where we plot the number-density profile of the boson star in the ground state for several initial values $\varphi_{0}$. The density profile is in the form of a plateau the length of which roughly measures the radius of the boson star. With decreasing $\varphi_{0}$, which corresponds to decreasing $G$, the plateau widens and becomes more pronounced. In the limit $G \rightarrow 0$, the boson star becomes an ordinary homogeneous condensate, with $\omega=m$.

We now turn to the study of nonzero temperature. As discussed in section 2, thermodynamic consistency strictly requires $\gamma=1$ which implies $\chi_{0} \leq 0$ owing to (68). It may be shown that if $\chi_{0} \leq 0$ and $\varphi_{0}>0$, then the function $\varphi(r)$ is monotonically increasing with $r$. To see this, note that near the origin the first derivative of $\varphi$ behaves as $\varphi^{\prime} \approx-\varphi_{0} \chi_{0} r / 3$, and hence, $\varphi$ increases for small $r$. Furthermore, at every point $r>0$ where $\varphi^{\prime}(r)=0$, the second derivative $\varphi^{\prime \prime}(r)$ is negative as a consequence of equation (55). This implies that the function $\varphi$ never reaches a maximum. Thus, equation (55) with (56)-(57) possesses no soliton solution if $\chi_{0} \leq 0$, and at any temperature $T>0$, the only physically acceptable solution, would be the trivial one $\varphi \equiv 0$. It seems that, in contrast to the self-gravitating fermion gas [21], a self-gravitating boson gas does not possess a nontrivial limiting configuration as $T \rightarrow 0$. Moreover, if we hold $N$ fixed and decrease the temperature sticking to $\gamma=1$, i.e., $\varphi \equiv 0, \mu$ will increase until we reach a limiting temperature $T_{\mathrm{c}}$ for which $\bar{\mu}(0)=m$. Further decrease of $T$ with fixed $N$ is no longer possible unless a condensation process takes place. If there were no gravity, we would decrease the temperature below $T_{\mathrm{c}}$ keeping $\mu=m$. As a result, the thermal density of particles would decrease and the density of particles in the ground state would adequately increase forming a homogeneous Bose-Einstein condensate $\varphi=$ const. In that process, the total number of particles would remain fixed.

In the presence of gravity, the formation of a homogeneous condensate is not possible since $\varphi=$ const is not a solution to (55). However, a condensation process will take place if below $T_{\mathrm{c}}$ the formation of a soliton is made possible by allowing $\chi_{0}>0$. This implies that the chemical potential at infinity $\mu$ is less than $\omega$, although the local chemical potential at 
the origin $\bar{\mu}(0)$ stays equal to $m$. The reason for this apparent thermodynamic inconsistency lies in the fact that general relativity does not allow configurations with asymptotically constant matter density. Of course, in the limit $G \rightarrow 0$, the soliton solution would become an ordinary homogeneous condensate, with $\mu=\omega=m$, as in the $T=0$ case discussed above. In numerical calculations we shall stick to the condition $\bar{\mu}(0)=m$ below $T_{\mathrm{c}}$. This condition, which prevents the Bose-Einstein distribution from becoming negative, fixes $\gamma=\sqrt{\chi_{0}+1}$ for $\chi_{0}>0$, and $\gamma=1$ for $\chi_{0} \leq 0$.

To proceed with the numerics, we have to specify the radius $R$. Although $R$ is an arbitrary parameter, the following physical considerations will give us its preferable order of magnitude. First, the size of the system is clearly much larger than the size that it would assume at zero temperature, i.e., $R$ must be larger than the radius of a boson star $R_{0} \sim 1 / \mathrm{m}$. Second, it should be smaller or at most of the order of the size it would take as a gas at high temperatures. To estimate the natural size of the purely gaseous phase, note that if the condensate vanishes, the quantities $\mathcal{M}$ and $r$ may be rescaled so that the dimensionless field equations remain in the same form as in (56)-(57) with $\tilde{\rho}$ and $\tilde{p}$ replaced by $\tilde{\rho}_{\text {th }}$ and $\tilde{p}_{\text {th }}$, respectively, and with $r$ and $\mathcal{M}$ measured in units of $M_{\mathrm{Pl}} / m^{2}$ and $M_{\mathrm{Pl}}^{3} / m^{2}$, respectively. Thus, the natural size of a purely gaseous phase is of the order $M_{\mathrm{Pl}} / m^{2}$ and therefore we expect the radius of our system to be in the range $1 / m \ll R \lesssim M_{\mathrm{Pl}} / m^{2}$.

Now suppose that at an early stage of the Universe evolution, when the temperature is high, say $T \gtrsim m$, a gravitating boson ensemble exists in a purely gaseous phase with the particle-number density of the order $n_{\mathrm{th}} \sim m^{3}$ within a volume of large radius $R_{\text {gas }}$. During the cooling down to temperatures $T \ll m$ the condensation will take place, in which a number of boson stars will be formed. During this evolution the particle number $N \sim R_{\text {gas }}^{3} m^{3}$ is conserved. Since a typical particle number in a boson star is of the order $1 / \eta$, the number of boson stars formed in the condensation process is approximately $n \sim \eta N \sim \eta R_{\text {gas }}^{3} m^{3}$ Therefore, the volume occupied by each of the boson stars is of the order $R_{\text {gas }}^{3} / n$ and the corresponding radius of the order

$$
R \sim \frac{R_{\text {gas }}}{n^{1 / 3}} \sim \frac{1}{\eta^{1 / 3} m}
$$

This quantity is much larger than the radius $R_{0}$ of the boson star itself, so the condensate will occupy only a small portion of the volume $V \sim R^{3}$. At $T \sim m$, the thermal contribution to the particle number is $N_{\text {th }} \sim R^{3} n_{\text {th }} \sim 1 / \eta$. Therefore, the contributions to the total charge of the thermal part and of the condensate are of the same order of magnitude despite the apparent incommensurability of the two densities in (62).

\section{$5 \quad$ Results and discussion}

To integrate equations (55)-(58) numerically, we take the radius of the system as $R=$ $\eta^{-1 / 3} m^{-1}$, in accordance with (71), and, for definiteness, we take the mass of the boson as $m=1 \mathrm{GeV}$. The choice of $m$ will practically not affect our results as long as $m \ll M_{\mathrm{Pl}}$.

For given $\alpha$, the initial values $\chi_{0}$ and $\varphi_{0}$ that yield the ground-state (zero-node) solution with the required particle number are not always uniquely determined. The reason is that, owing to general-relativistic effects, there exists a finite $\chi_{0}$ for which $N$ and $M$ reach a 
maximum. This limiting configuration is similar to the Oppenheimer-Volkoff limit for the degenerate fermion stars. Further increase of $\chi_{0}$ makes $N$ oscillate about a nonzero limiting value $N_{\infty}$ that corresponds to the infinite central density. In Fig. \& we plot the particle number $N$ versus $\chi_{0}$ for various $\alpha$, including $\alpha=\infty$ which corresponds to zero temperature. It is clear that for each $\alpha$ there will be at least two configurations with the same $N$, if $N$ is slightly below the maximum. However, the configurations represented by the points on the right of the maximum on each curve are thermodynamically unstable since their free energy, as we shall shortly see, is larger than the free energy of the corresponding configurations on the left of the maximum. Therefore, if we restrict our consideration to stable configurations, the quantities $\chi_{0}$ and $\varphi_{0}$ will be uniquely determined.

Fixing $N=0.5$, which is below the maximum of the zero temperature curve, we now plot the initial values $\chi_{0}$ and $\varphi_{0}$ as functions of inverse $\alpha$ in Figs. 3 and 4 , respectively. At the critical point $1 / \alpha_{\mathrm{c}}=T_{\mathrm{c}} / m=0.582, \chi_{0}$ changes the sign and $\varphi_{0}$ vanishes. Thus, the configurations for which $\alpha<\alpha_{\mathrm{c}}$ are purely thermal and those with $\alpha>\alpha_{\mathrm{c}}$ are mixed. For each $\alpha$ we can find $\omega, \mu$, and $T$ by making use of equations (54) and (69).

In Fig. 5 we plot the free energy per particle, calculated using (47) and (48), as a function of temperature. The full line represents the free energy of the configurations corresponding to the points on the left of the maxima in Fig. 2, whereas the dashed line represents the free energy corresponding to the points on the right of the maxima. As expected, the latter free energy is everywhere larger than the former, hence, the configurations it represents are thermodynamically unstable. In what follows we restrict our attention to stable configurations only. The critical temperature $T_{\mathrm{c}}=0.582 \mathrm{~m}$ is indicated by a vertical line. In Figs. 6 and 0 the entropy per particle and the total mass are plotted as functions of temperature. Since $S$ and $M$ are both continuous functions at $T_{\mathrm{c}}$, we conclude that the process of condensation is a second-order phase transition with the naturally defined order parameter $\varphi_{0}$ plotted in

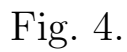

In order to facilitate the physical understanding of our results, let us mention the properties of the particular soliton configuration in physical units. The radius of the soliton at $T=0$ is a few times larger than $(\mathrm{cm} / \hbar)^{-1}$, so for $m=1 \mathrm{GeV}$ it is approximately $1.2 \times 10^{-12}$ $\mathrm{cm}$, whereas the radius of the whole star at $T \neq 0$ is $1.04 \mathrm{~cm}$. The total mass of the soliton at $T=0$ is $M=1.3 \times 10^{11} \mathrm{~kg}$, the particle number $N=7.5 \times 10^{37}$, and the critical temperature $T_{c}=0.582 \mathrm{GeV}$.

At this stage it is instructive to compare our results with the approximate analytical result of a corresponding free-boson system. Taking the lowest contributions in the hightemperature expansion, one can find simple analytical expressions that describe properties of the free boson gas [3]. In particular, the critical temperature is given by

$$
T_{\mathrm{c}}=\left(\frac{3 N}{m V}\right)^{1 / 2}
$$

Using $V=4 \pi R^{3} / 3$ we find

$$
\frac{T_{\mathrm{c}}}{m}=\frac{3}{2 \sqrt{\pi}} R^{-3 / 2} N^{1 / 2},
$$

with $R$ measured in units of $\eta^{-1 / 3} m^{-1}$ and $N$ in units of $\eta^{-1}$. Within the same approximation, 
the temperature dependence of the chemical potential is given by

$$
\frac{\mu}{m}=\left(\frac{T_{\mathrm{c}}}{T}\right)^{2}
$$

for $T \geq T_{\mathrm{c}} \gg m$, and $\mu=m$ for temperatures $T \leq T_{\mathrm{c}}$.

In Figs. 8 and 9 we plot the chemical potential $\mu$ at infinity and the chemical potential $\bar{\mu}(0)$ at the origin, respectively, as functions of temperature. For comparison, in Fig. 8 we also give the discussed approximate analytical result for a free boson system. It is worth noting the following two effects. First, the critical temperature is almost exactly equal to that obtained for a free relativistic Bose system. Second, the functions describing the temperature dependence of $\mu$ and $\bar{\mu}(0)$ for $T>T_{\mathrm{c}}$ are almost exactly the same. The reason for both effects is that $g_{00}(r)$ in a purely gaseous phase is practically constant on the interval $(0, R)$. The departure of the analytical result for a free boson system from the numerical result for a gravitating boson system is a general-relativistic effect below $T_{\mathrm{c}}$, and above $T_{\mathrm{c}}$ is just due to the high-temperature approximation.

Our results depicted in Figs. 2-9 depend, of course, on the choice of $R$ and $N$. However, as in the case of a free gas, the critical temperature should not depend on $R$ nor on $N$ if the average density is kept fixed. We have checked that, if we keep the ratio $N / R^{3}$ fixed, our $T_{\mathrm{c}}$ remains constant to very high accuracy with $R$ varying by several orders of magnitude.

So far, we have studied the condensation process assuming $m \ll M_{\mathrm{Pl}}$, i.e., $\eta \ll 1$. Now, let us investigate an extreme general-relativistic case when the value of the boson mass is a sizable fraction of the Planck mass, say $m=0.1 M_{\mathrm{Pl}}$, yielding $\eta=0.01$. In this case, the natural length scale $\eta^{-1 / 2} m^{-1}$ of the purely thermal phase becomes comparable with the natural size $\eta^{-1 / 3} m^{-1}$ of the mixed phase, and we expect large gravitational effects.

The calculations are presented in Figs. 10 and 11 in which, respectively, the free energy and entropy are shown as functions of temperature for $\eta=0.01$ and $R=10 \eta^{-1 / 2} m^{-1}$. The part of the curve in Fig. 10 that starts from $T=0$, makes the loop, and ends at the sharp cusp, represents the mixed soliton-gas phase. The rest of the curve, which can be continuously extended to high temperatures, represents the purely gaseous phase. In the temperature interval $T=(0.003-0.015) m$ there are three distinct solutions of which only two are physical, namely, those for which the free energy assumes a minimum. The cusp corresponds to the naive transition point. However, the actual transition takes place at the temperature $T_{\mathrm{c}}$, where the free energy of the mixed phase and that of the gas become equal. Hence, it is a first-order phase transition. The dotted curves in Figs. 10 and 11 represent the physically unstable solution. In our example, the transition temperature is $T_{c}=0.00607 \mathrm{~m}$, as indicated in the plots by the dashed line. The latent heat per particle released during the phase transition is given by the entropy difference at the point of discontinuity

$$
\frac{\Delta M}{N}=\frac{\Delta S}{N} T_{\mathrm{c}}=0.0206 m
$$

This phase transition is similar to the gravitational phase transition in self-gravitating fermionic systems [21], with one important distinction: in a gravitating bosonic system the phase transition is first order only in the extreme general-relativistic regime, whereas in gravitating fermionic systems it remains first order even in the Newtonian regime [22, 23]. 


\section{Conclusions}

In this work, we have studied a canonical system of self-gravitating bosons at finite temperature in a general-relativistic framework. We have numerically solved the system of self-consistency equations consisting of a Klein-Gordon equation coupled to Einstein field equations and the equation of state for a gravitating boson gas. We have investigated the circumstances under which this system undergoes a Bose-Einstein condensation. This condensation is quite distinct from the usual one in that it involves the formation of a soliton with the spherically symmetric matter distribution concentrated around the origin, as opposed to the usual spatially homogeneous Bose-Einstein condensate. In the $T \rightarrow 0$ limit, the soliton becomes a mini boson star. The condensation begins with a second order phase transition at the critical temperature $T_{\mathrm{c}}$. The system exists in two phases: a gaseous phase above $T_{\mathrm{c}}$ and the mixed soliton-gas phase below $T_{c}$. The critical temperature is approximately proportional to the square root of the average particle-number density and is very close the the corresponding critical temperature of a free boson gas.

General relativistic effects become important when the boson mass is a few orders of magnitude away from the Planck mass. In that case, the condensation begins with a first-order

phase transition that qualitatively resembles the gravitational phase transition of fermionic matter.

\section{Acknowledgments}

This work was supported by the Ministry of Science and Technology of the Republic of Croatia under Contract No. 00980102.

\section{References}

[1] J.I. Kapusta, Phys. Rev. D 24 (1981) 426.

[2] H.E. Haber and H.A. Weldon, Phys. Rev. Lett. 46 (1981) 1497.

[3] H.E. Haber and H. A. Weldon, Phys. Rev. D 25 (1982) 502.

[4] R. Friedberg, T.D. Lee, and A. Sirlin, Phys. Rev. D 13 (1976) 2739.

[5] S. Coleman, Nucl. Phys. B 262 (1985) 263.

[6] D.J. Kaup, Phys. Rev. 172 (1968) 1331.

[7] R. Friedberg, T.D. Lee and Y. Pang, Phys. Rev. D 35 (1987) 3640.

[8] P. Jetzer, Phys. Rep. 220 (1992) 163.

[9] E. Seidel and W.-M. Suen, Phys. Rev. Lett. 72 (1994) 2516.

[10] E.W. Mielke and F.E. Schunck, Nucl. Phys. B 564 (2000) 185. 
[11] G. Ingrosso and R. Ruffini, Nuovo Cim. B 101 (1988) 369.

[12] M. Laine and M. Shapshnikov, Nucl. Phys. B 532 (1998) 376.

[13] G.W. Gibbons and S.W. Hawking Phys. Rev. D 15 (1977) 2752.

[14] S.W. Hawking, The path-integral approach to quantum gravity, in General relativity: An Einstein centenary survey, edited by S.W. Hawking and W. Israel (Cambridge University Press, Cambridge, 1979).

[15] J. I. Kapusta, Finite-temperature field theory (Cambridge University Press, Cambridge 1989).

[16] R.C. Tolman, Relativity, thermodynamics and cosmology (Clarendon, Oxford, 1934).

[17] L.D. Landau and E.M. Lifshitz, Fluid mechanics (Pergamon, Oxford, 1959).

[18] J. Ehlers, Survey of general relativity theory, in Relativity, astrophysics and cosmology, edited by W. Israel (D. Reidel, Dordrecht/Boston, 1973).

[19] J.M. Bardeen, B. Carter and S.W. Hawking, Commun. Math. Phys. 31 (1973) 161.

[20] N. Bilić and R.D. Viollier, Gen. Rel. Grav. 31 (1999) 1105.

[21] N. Bilić and R.D. Viollier, Eur. Phys. J. C 11 (1999) 173.

[22] J. Messer, J. Math. Phys. 22 (1981) 2910.

[23] N. Bilić and R.D. Viollier, Phys. Lett. B 408 (1997) 75;

N. Bilić and R.D. Viollier, Nucl. Phys. B (Proc. Suppl.) 66 (1998) 256. 


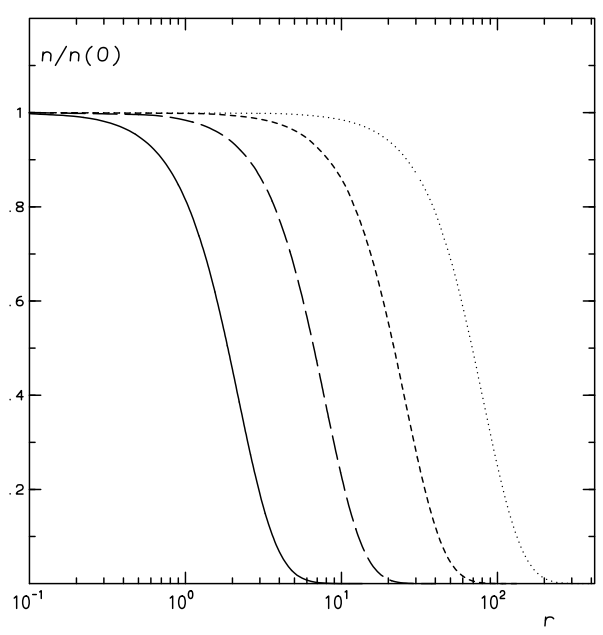

Figure 1: Normalized particle-number density in a boson star at $T=0$ for $\varphi_{0}$ equal to 0.0764 (full line), 0.01 (long dashed line), 0.001 (short dashed line), and 0.0001 (dotted line). The radius $r$ is in units of $m^{-1}$

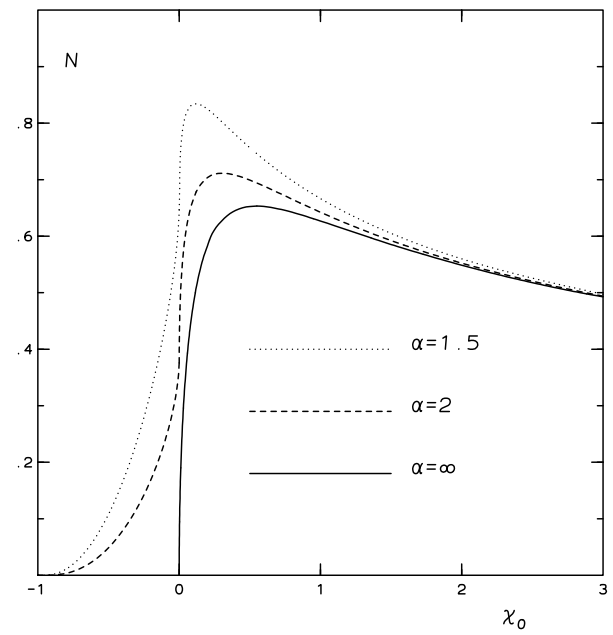

Figure 2: Particle number $N$ (in units of $M_{\mathrm{Pl}}^{2} / m^{2}$ ), versus $\chi_{0}$ for various $\alpha$. 


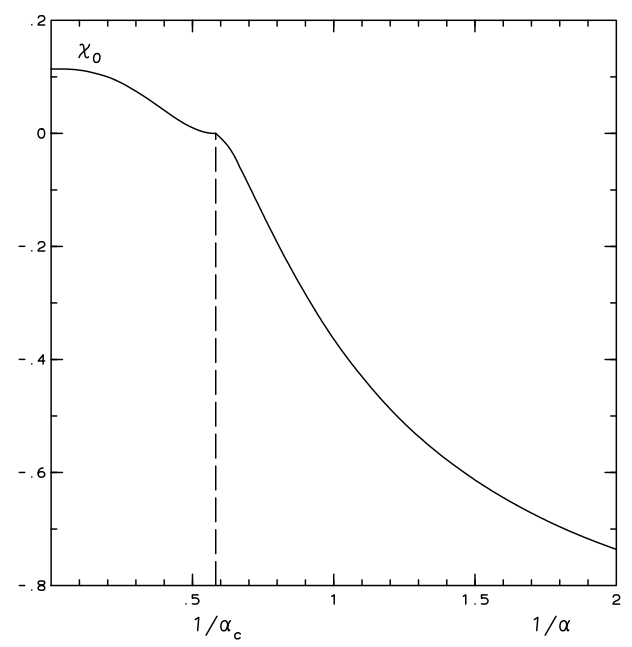

Figure 3: Initial value of the metric field $\chi_{0}$ that yields the zero-node soliton solution with $N=0.5 M_{\mathrm{Pl}}^{2} / m^{2}$, within a sphere of radius $R=\left(M_{\mathrm{Pl}} / m\right)^{2 / 3} m^{-1}$, versus $1 / \alpha$.

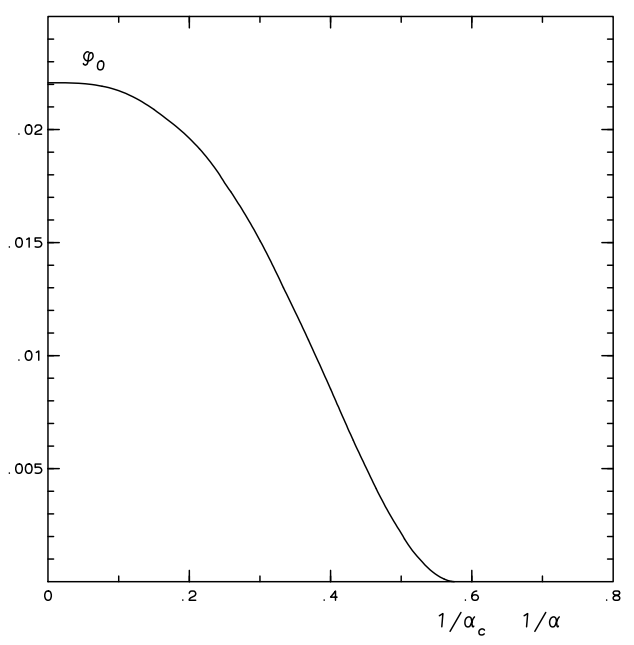

Figure 4: Initial value $\varphi_{0}$ as a function of $1 / \alpha$ for $N$ and $R$ as in Fig. 3 . 


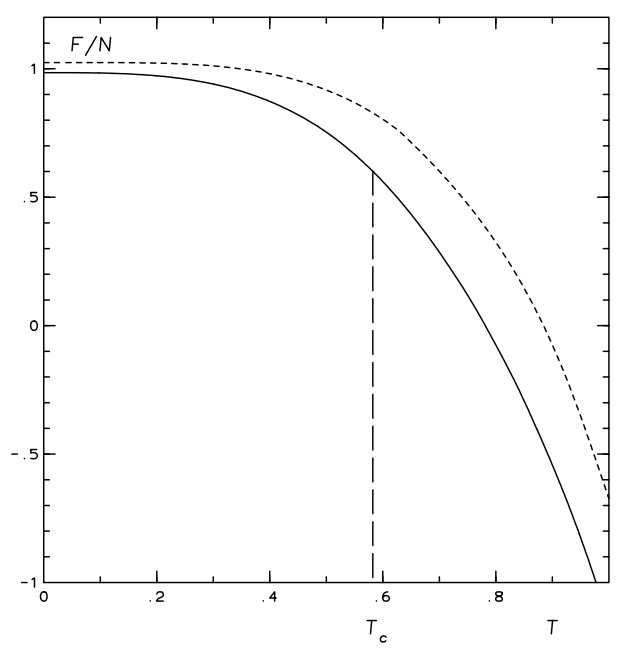

Figure 5: Free energy per particle $F / N$ (in units of $m$ ) versus temperature T (in units of $m$ ) for $N$ and $R$ as in Fig. 3 .

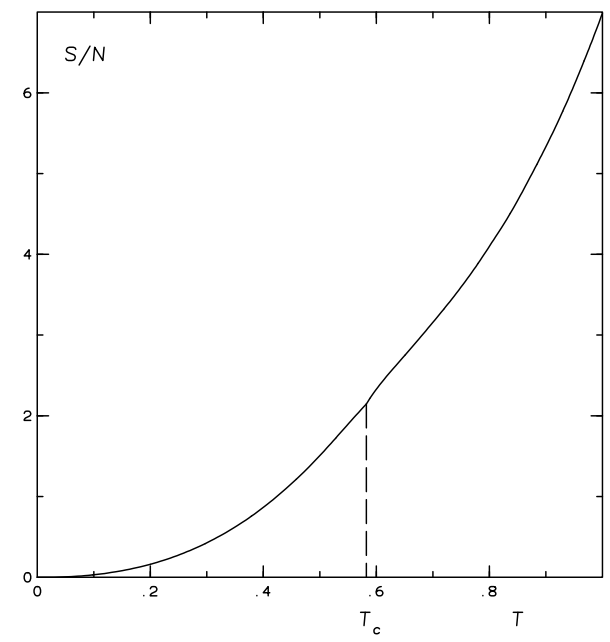

Figure 6: Entropy per particle versus temperature (in units of $m$ ) for $N$ and $R$ as in Fig. 3 . 


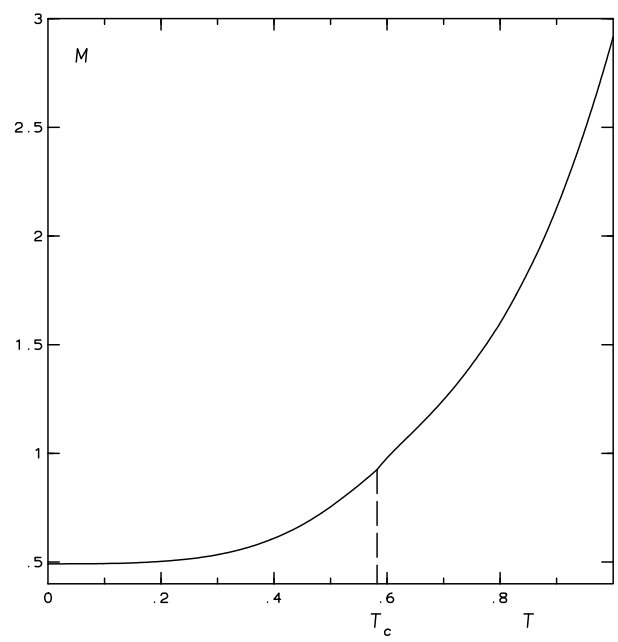

Figure 7: Total mass (in units of $M_{\mathrm{Pl}}^{2} / m$ ) versus temperature (in units of $m$ ) for $N$ and $R$ as in Fig. 3.

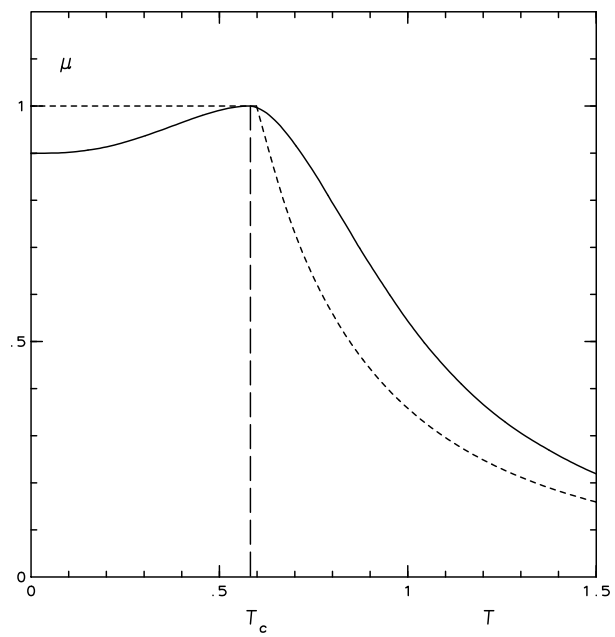

Figure 8: Chemical potential $\mu$ (in units of $m$ ) at $r=\infty$ as a function of temperature (in units of $m$ ) for gravitating bosons (solid line) and for free bosons in the high-temperature approximation (dashed line) for $N$ and $R$ as in Fig. 3. 


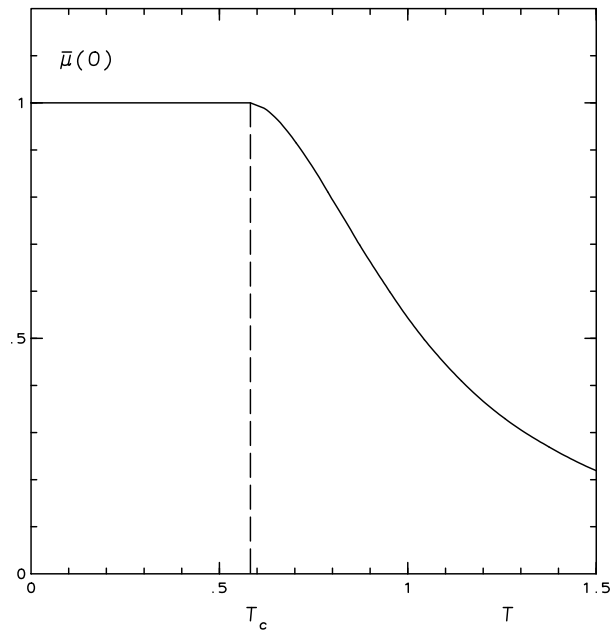

Figure 9: Chemical potential $\bar{\mu}$ (in units of $m$ ) at $r=0$ as a function of temperature (in units of $m$ ) for $N$ and $R$ as in Fig. 3 .

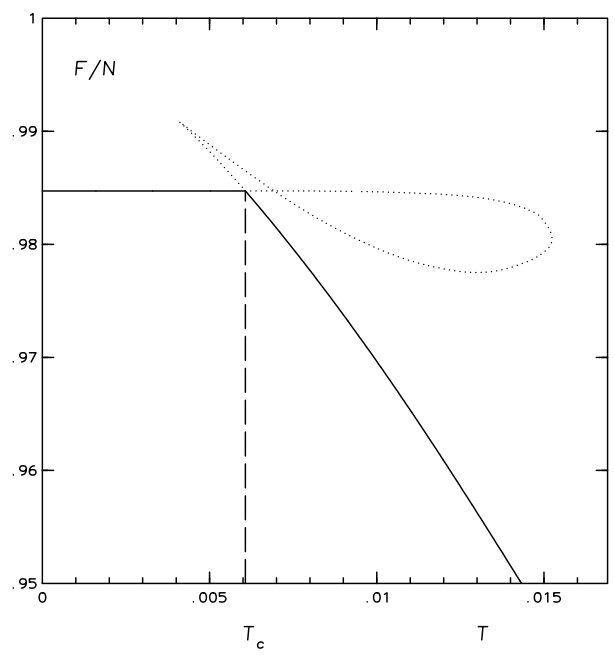

Figure 10: Free energy per particle (in units of $m$ ) versus temperature (in units of $m$ ) for $\eta \equiv m^{2} / M_{\mathrm{Pl}}^{2}=0.01$ and $N=0.5 M_{\mathrm{Pl}}^{2} / m^{2}$. 


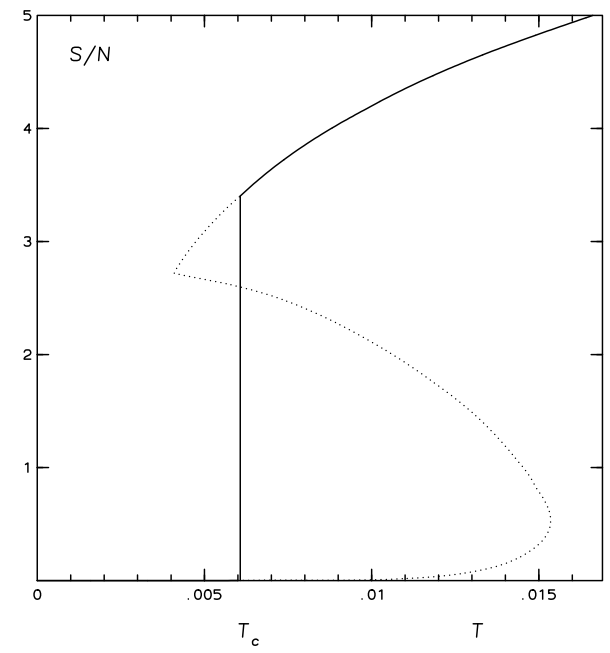

Figure 11: Entropy per particle versus temperature (in units of $m$ ) for $\eta \equiv m^{2} / M_{\mathrm{Pl}}^{2}=0.01$ and $N=0.5 M_{\mathrm{Pl}}^{2} / \mathrm{m}^{2}$. 